\title{
Holographic dark energy in Brans-Dicke cosmology with chameleon scalar field
}

\author{
M. R. Setare ${ }^{1, *}$ and Mubasher Jamil ${ }^{2, \dagger}$ \\ ${ }^{1}$ Department of Science of Bijar, University of Kurdistan, Bijar, Iran \\ 2 Center for Advanced Mathematics and Physics, \\ National University of Sciences and Technology, H-12, Islamabad, Pakistan
}

\begin{abstract}
We study a cosmological implication of holographic dark energy in the Brans-Dicke gravity. We employ the holographic model of dark energy to obtain the equation of state for the holographic energy density in non-flat (closed) universe enclosed by the event horizon measured from the sphere of horizon named $L$. Our analysis shows that one can obtain the phantom crossing scenario if the model parameter $\alpha$ (of order unity) is tuned accordingly. Moreover, this behavior is achieved by treating the Brans-Dicke scalar field as a Chameleon scalar field and taking a nonminimal coupling of the scalar field with matter. Hence one can generate phantomlike equation of state from a holographic dark energy model in non-flat universe in the Brans-Dicke cosmology framework.
\end{abstract}

*Electronic address: rezakord@ipm.ir

$\dagger$ Electronic address: mjamil@camp.nust.edu.pk 


\section{INTRODUCTION}

Many cosmological observations, such as SNe Ia [1], WMAP [2], SDSS [3], Chandra Xray observatory [4], etc., reveal that our universe is undergoing an accelerating expansion. To explain this cosmic positive acceleration, mysterious dark energy has been proposed. There are several dark energy models which can be distinguished by, for instance, their equation of state $(\mathrm{EoS})\left(w_{\Lambda}=\frac{P_{\Lambda}}{\rho_{\Lambda}}\right)$ during the evolution of the universe. Astrophysical data also indicate that $w_{\Lambda}$ lies in a very narrow strip close to -1 . The case $w_{\Lambda}=-1$ corresponds to the cosmological constant. For $w_{\Lambda}$ less than -1 the phantom dark energy [5] is observed, and for $w_{\Lambda}$ more than -1 (but less than $\frac{-1}{3}$ ) the dark energy is described by quintessence [6]. More ever, the analysis of the properties of dark energy from recent observational data mildly favor models of dark energy with $\omega$ crossing -1 line in the near past. So, the phantom phase equation of state with $\omega_{\Lambda}<-1$ is still mildly allowed by observations. Most of dark energy models treat scalar field(s) as dark component(s) with a dynamical equation of state. So far, a large class of scalar-field dark energy models have been studied, including quintessence [6], K-essence [7], tachyon [8], phantom [5], ghost condensate $[9,10]$ and quintom [11], and so forth. The introduction of a scalar field potential augmented by a scalar field dependent coupling constant solved many problems and provided clues to the solutions of many of the outstanding cosmological problems in particular within the framework of the Brans-Dicke (BD) cosmology.

In recent years, the holographic dark energy has been studied as a possible candidate for dark energy. It is motivated from the holographic principle which might lead to the quantum gravity to explain the events involving high energy scale. In the thermodynamics of black hole, there is a maximum entropy in a box of length $L$, commonly termed, the Bekenstein-Hawking entropy bound $S \sim M_{p}^{2} L^{2}(c=\hbar=1)$, which scales as the area of the box $A \sim L^{2}[12]$. To avoid the breakdown of the local quantum field theory, Cohen et al [13] suggested that the entropy for an effective field theory should satisfy $L^{3} \Lambda^{3} \leq S^{3 / 4} \sim\left(M_{p} L\right)^{3 / 2}$. Here $L$ is the size of the region which serves as the infra-red cut-off while $\Lambda$ is the ultra-violet cut-off. Incidently this last equation can be re-written in the form $L^{3} \rho_{\Lambda} \leq L M_{p}^{2}$, where $\rho_{\Lambda} \sim \Lambda^{4}$ is the energy density corresponding to the zero-point energy and cut-off $\Lambda$. Thus the total energy in a region of size $L$ cannot exceed the mass of a black hole of the same size. From this discussion we can deduce that $\rho_{\Lambda} \leq M_{p}^{2} L^{-2}$. This inequality can be saturated and it becomes $\rho_{\Lambda}=3 c^{2} M_{p}^{2} L^{-2}$, where $3 c^{2}$ is introduced for convenience.

It is well-known that general relativity although performs fairly well at the solar system scale but fails at the cosmic scales at which it is unable to explain the origin of dark energy or present accelerated cosmic expansion. This has lead scientists to propose alternative theories of gravity. Some of these gravity theories are actually modifications of Einstein's general relativity including scalar-tensor gravity [14] and $f(R)$ gravity [15]. The earliest modifications to Einstein's gravity was the Kaluza-Klein gravity [16] which was intended to unify gravity with the electromagnetic force. In the early 1960s, Brans and Dicke proposed a relativistic theory of gravity based on the Einstein's theory of general relativity [17]. This was the first gravity theory in which the dynamics of gravity were described by a scalar field while spacetime dynamics were represented by the metric tensor. Later on it turned out that the theory can pass the experiments from the solar system [18]. An attractive feature of BD theory is that the scalar field is a fundamental element of the theory, quite contrary to other models in which scalar field is introduced separately in an ad hoc manner. The basic notion is that the BD scalar field plays the role of 
quintessence or K-essence and lead to cosmological acceleration. However it happens only in certain cases: for instance the cosmic acceleration is permissible if the BD parameter is constrained in the range $-2<w<-3 / 2$, which not only violate the energy conditions on the scalar field but are also inconsistent with a radiation dominated epoch, unless $w$ is a time dependent parameter.

As discussed earlier that the scalar field plays the role of dark energy in the BD gravity. In this connection, we can relate the energy density of holographic dark energy (in fact, any other form of dark energy in general) and the BD scalar field in a consistent way. The holographic dark energy in the framework of Brans-Dicke gravity has been investigated in $[19,20]$. We consider a Brans-Dicke framework in which there is a non-minimal coupling between the scalar field and the background geometry. The action is modified due to the inclusion of a non-minimal coupling of the scalar field with the matter. This work is actually motivated by the work of Clifton and Barrow [21] where they investigated the behavior of an isotropic cosmological model in the early as well as in the late time limits in the Brans-Dicke framework. However, the non-minimal coupling of a scalar field with both of geometry and the matter sector has been in use for quite a long time, for instance, in the dilaton gravity [22].

\section{HOLOGRAPHIC ENERGY DENSITY IN BRANS-DICKE FRAMEWORK}

We start with the action for the Brans-Dicke theory [23]

$$
A=\int \sqrt{-g} d^{4} x\left[-\Phi R+\frac{\omega}{\Phi} \Phi^{, \mu} \Phi_{, \mu}+f(\Phi) L_{m}\right],
$$

in the Jordan frame. Here $R$ is the Ricci scalar and $\Phi=\Phi(t)$ is the Brans-Dicke scalar field representing the inverse of Newton's constant which is allowed to vary with space and time and $\omega$ is the generic dimensionless parameter of the Brans-Dicke theory. The matter Lagrangian $L_{m}$ represents the perfect fluid matter. Notice that taking $\omega=0$ and $f=0$ in (1) yields the Einstein-Hilbert action. In the Jordan frame, the matter minimally couples to the metric and there is no interaction between the scalar field $\Phi$ and the matter field [24]. Here $\omega$ is an unknown parameter.

We assume the background to be Friedmann-Robertson-Walker (FRW) spacetime

$$
d s^{2}=-d t^{2}+a(t)^{2}\left[\frac{d r^{2}}{1-k r^{2}}+r^{2}\left(d \theta^{2}+\sin ^{2} \theta d \phi^{2}\right)\right] .
$$

Here $a$ is the scale factor while $k=-1,0,+1$ corresponds to open, flat and closed universe. The gravitational field equations derived from the variation of the action (1) with respect to FRW metric is

$$
\begin{aligned}
H^{2}+\frac{k}{a^{2}} & =\frac{f}{3 \Phi} \rho+\frac{1}{6} \omega \frac{\dot{\Phi}^{2}}{\Phi^{2}}-H \frac{\dot{\Phi}}{\Phi}, \\
2 \frac{\ddot{a}}{a}+H^{2}+\frac{k}{a^{2}} & =p-\frac{\omega}{2} \frac{\dot{\Phi}^{2}}{\Phi^{2}}-\frac{\ddot{\Phi}}{\Phi}-2 H \frac{\dot{\Phi}}{\Phi} .
\end{aligned}
$$

Here total energy density $\rho=\rho_{\Lambda}+\rho_{m}$, the sum of energy densities of holographic dark energy and matter. Also the total pressure $p=p_{\Lambda}+p_{m}$, is the sum of pressure of holographic dark energy and matter. The dynamical equation for the scalar field is

$$
6\left(\frac{\ddot{a}}{a}+H^{2}+\frac{k}{a^{2}}\right)+\omega \frac{\dot{\Phi}^{2}}{\Phi^{2}}+L_{m} f_{, \Phi}-2 \omega \frac{\ddot{\Phi}}{\Phi}=0 .
$$


The parameter $\omega$ that appears in the above field equations (3) to (5) can be observed astronomically. The Cassini experiment implies that $\omega>10^{4}[25]$. We define parameters as

$$
\Omega_{k}=\frac{2 k}{a^{2} H^{2}}, \quad \rho_{c r}=6 \Phi H^{2} .
$$

The holographic dark energy is related to the Brans-Dicke scalar field by

$$
\rho_{\Lambda}=6 \Phi L^{-2}
$$

where the holographic parameter is fixed at $c=1$. Since there are numerous candidates of dark energy, it is important to know how these candidates are related to each other. Notice that from Eq. (7), the dynamics of BD scalar field are connected with that of the energy density of HDE. Also note that if the infrared cut-off is taken as the Hubble horizon then the energy density of HDE and critical density match identically. This situation generically arises in inflation scenario where $L=H^{-1}$ (a constant length scale). However in the overall evolution of the Universe with $0<c<1$ and $L \neq H^{-1}$, the two energy densities are not the same and $\rho_{\Lambda}<\rho_{c r}$.

There are various choices of infrared cut-off for the cosmological length scale available in the literature. For instance, the simplest choice for infrared cut-off is the Hubble horizon $H^{-1}$, but Hsu [26] has shown that in this case the EoS parameter $\omega_{\Lambda}=0$, which can not describe the cosmic acceleration. Another suggestion is the particle horizon $\left(L_{p}=a(t) \int_{0}^{t} \frac{d t^{\prime}}{a\left(t^{\prime}\right)}\right)$, but it turns out that $\omega_{\Lambda}>-1 / 3$, a form of exotic matter that is not suitable to derive acceleration. A suitable choice of infrared cut-off was then suggested by $\mathrm{Li}[27]$ and is termed as the future event horizon (defined below). Note that the choice of horizon (or the infrared cut-off) is independent of the spatial curvature of FRW spacetime. According to the formulation of holographic dark energy in non-flat geometry, the cosmological length $L$ is considered to be:

$$
L \equiv \frac{a(t)}{\sqrt{|k|}} \operatorname{sinn}(\sqrt{|k|} y), \quad y=\frac{R_{h}}{a(t)},
$$

where $R_{h}$ is the future event horizon defined by

$$
R_{h}(a)=a \int_{t}^{\infty} \frac{d t^{\prime}}{a\left(t^{\prime}\right)}=a \int_{a}^{\infty} \frac{d a^{\prime}}{H a^{\prime 2}},
$$

and

$$
\frac{1}{\sqrt{|k|}} \operatorname{sinn}(\sqrt{|k|} y)= \begin{cases}\sin y & k=+1 \\ y & k=0, \\ \sinh y & k=-1\end{cases}
$$

In some recent studies, some new infrared cut-offs have been proposed. In [28], the authors have added the square of the Hubble parameter and its time derivative within the definition of holographic dark energy. While in [29], the authors propose a linear combination of particle horizon and the future event horizon. However, in this paper we will adopt Li's proposal.

The holographic dark energy satisfies

$$
\Omega_{\Lambda}=\frac{1}{H^{2} L^{2}} .
$$


Differentiating (8), we obtain

$$
\dot{L}=\frac{1}{\sqrt{\Omega_{\Lambda}}}-\operatorname{cosn}(\sqrt{|k|} y)
$$

where

$$
\operatorname{cosn}(\sqrt{|k|} y)= \begin{cases}\cos y & k=+1 \\ 1 & k=0 \\ \cosh y & k=-1\end{cases}
$$

The energy conservation equation is

$$
\dot{\rho}_{\Lambda}+3 H\left(\rho_{\Lambda}+p_{\Lambda}\right)=0
$$

where $p_{\Lambda}$ is the pressure of the HDE. Notice that the above expression (14) for HDE is valid $[19,30]$ while the continuity equation for matter is given in [23]. Differentiating (7) w.r.t. time, we get

$$
\dot{\rho}_{\Lambda}=\rho_{\Lambda}\left(\frac{\dot{\Phi}}{\Phi}-2 \frac{\dot{L}}{L}\right) .
$$

Taking an ansatz for BD scalar field [23]

$$
\frac{\dot{\Phi}}{\Phi}=-\frac{\alpha}{H},
$$

where $\alpha$ is a constant of order unity (and not arbitrary). There is no a priori physical motivation for this choice, this is purely phenomenological which leads to the desired behavior of the deceleration parameter $q$ of attaining a negative value at the present epoch from a positive value during a recent past. Using (12) and (16) in (15), we obtain

$$
\dot{\rho}_{\Lambda}=-\rho_{\Lambda} H\left[\frac{\alpha}{H^{2}}+2\left(1-\sqrt{\Omega_{\Lambda}} \operatorname{cosn}(\sqrt{|k|} y)\right)\right] .
$$

Putting (17) in (14), we get

$$
p_{\Lambda}=-\frac{1}{3}\left[1-\frac{\alpha}{H^{2}}+2 \sqrt{\Omega_{\Lambda}} \operatorname{cosn}(\sqrt{|k|} y)\right] \rho_{\Lambda} .
$$

Thus equation of state parameter of dark energy in Brans-Dicke cosmology becomes

$$
\omega_{\Lambda}=-\frac{1}{3}\left[1-\frac{\alpha}{H^{2}}+2 \sqrt{\Omega_{\Lambda}} \operatorname{cosn}(\sqrt{|k|} y)\right]
$$

Notice that for particular choices of $\alpha$, it is possible to attain $\omega_{\Lambda}>-1, \omega_{\Lambda}<-1$ and values in between. Thus a phantom crossing scenario can be constructed in the present model.

Now we determine the evolution of $\Omega_{\Lambda}$. We differentiate it w.r.t $t$ to get

$$
\frac{d \Omega_{\Lambda}}{d t}=-2 \Omega_{\Lambda}\left(\frac{\dot{H}}{H}+\frac{\dot{L}}{L}\right) .
$$

Using the definitions

$$
\dot{H}=-H^{2}(1+q), \quad q=-\frac{\ddot{a}}{a H^{2}}
$$


in Eq. (20), we obtain

$$
\frac{d \Omega_{\Lambda}}{d t}=2 \Omega_{\Lambda} H\left[q+\sqrt{\Omega_{\Lambda}} \operatorname{cosn}(\sqrt{|k|} y)\right] .
$$

Here $q$ is the deceleration parameter. From Eq. (4), it is easy to check

$$
q=\frac{1}{\alpha+2 H^{2}}\left[H^{2}\left(1+\frac{\Omega_{k}}{2}\right)+\frac{\alpha^{2}}{H^{2}}\left(1+\frac{\omega}{2}\right)-3 \alpha-p\right] .
$$

Notice that if we take $k=0$ and $p=0$, then (23) reduces to the case discussed by Das and Banerjee [23], thus our paper extends their work.

In a spatially flat universe, we have

$$
\omega_{\Lambda}=-\frac{1}{3}\left(1-\frac{\alpha}{H^{2}}+2 \sqrt{\Omega_{\Lambda}}\right) .
$$

Using $\Omega_{\Lambda}=0.73$ in (24), we notice that $\omega_{\Lambda}>-1$ when $\alpha>-0.2912 H^{2}$, while $\omega_{\Lambda}<-1$ when $\alpha<-0.2912 H^{2}$.

To check how the HDE state parameter $\omega_{\Lambda}$ evolves in a spatially flat Universe, we calculate its time derivative and get

$$
\dot{\omega}_{\Lambda}(t)=\frac{2}{3 H}(1+q)-\frac{2}{3} H \sqrt{\Omega_{\Lambda}}\left(q+\sqrt{\Omega_{\Lambda}}\right) .
$$

Notice that in an accelerating Universe, $q \leq-1$, therefore (25) implies $\dot{\omega}_{\Lambda}<0$. Hence in HDE dominated flat Universe, the state parameter evolves gradually to super-negative values.

In the end, we would comment that the present study can also be performed for the new agegraphic dark energy (NADE). In NADE, the infrared cut-off is the conformal

time, $\eta=\int_{0}^{a} \frac{d a^{\prime}}{H a^{\prime 2}}$, i.e. the age of the Universe is used as a parameter in the model [31]. In this context, it will be interesting to see whether the phantom crossing will take place in both flat and non-flat FRW backgrounds in the context of BD cosmology. However there are several disadvantages with the agegraphic dark energy: it can not generate the inflation era in the early universe unlike the holographic dark energy; it cannot produce a phantom dominated universe since its equation of state parameter is always greater than -1 ; its energy density decreases with time unlike any other dark energy candidate; quantum corrections are generally ignorable and it worse fits with the observational data [32, 33]. From the same study [32], it is deduced that the observational data is wellfitted with the dynamical dark energy models with only one free parameter including the holographic dark energy model. However the NADE model is completely disfavored by the astrophysical data. Thus our present study is also consistent with the empirical findings.

\section{CONCLUSION}

In this paper, we have studied holographic dark energy in the Brans-Dicke cosmological model. The later model contains a dynamical time dependent scalar field that behaves like dark energy and produces cosmic acceleration. Following the work of [23], we assumed 
the scalar field to be chameleon i.e. a scalar field interacting with the matter. The two entities are connected to each other via a coupling parameter $f$. Although we took $f$ to be arbitrary function, Das and Banerjee [23] took few ansatz for it and studied the behavior of the model. Ideally, the coupling between dark energy (of any type including the BD scalar field) and matter should be derived from a theory of quantum gravity. In the absence of such a theory, we decided to keep our analysis general regardless of the specification of $f$. The coupling parameter could also be constrained from the astrophysical data.

Earlier it had been observed in [19] that if the BD scalar field is minimally coupled to gravity then phantom crossing is not possible. In that model, the author took the BD scalar field as related with the HDE. The study of [19] motivated us to consider a modification of the BD framework by introducing a chameleon scalar field interacting with the matter. By establishing a correspondence between the BD scalar field and the HDE, we have found that phantom crossing is possible by tuning the free parameter $\alpha$. Notice that the parameter $\alpha$ is not arbitrary but is a constant of order unity. We would also comment that the fine tuning of parameters is a general feature of almost every cosmological model and our model is also no exception. Finally, we took the infrared cut-off of the cosmological spacetime to be future event horizon $R_{h}$ and performed the analysis in both flat and non-flat backgrounds.

\section{Acknowledgment}

Both of us would like to thank the referee for his critical comments to improve this work.

[1] A.G. Riess et al, Astrophys. J. 607, 665 (2004); R.A. Knop et al, Astrophys. J. 598, 102 (2003); S. Perlmutter et al, Astrophys. J. 517, 565 (1999).

[2] C. L. Bennett et al, Astrophys. J. Suppl. 148, 1 (2003); D. N. Spergel et al, Astrophys. J. Suppl. 148, 175 (2003).

[3] M. Tegmark et al, Phys. Rev. D 69, 103501 (2004); U. Seljak et al, Phys. Rev. D 71, 103515 (2005); J. K. A-M. et al, astro-ph/0507711.

[4] S. W. Allen et al, Mon. Not. Roy. Astron. Soc. 353, 457 (2004).

[5] R.R. Caldwell, Phys. Lett. B 545, 23 (2002); S. Nojiri and S. D. Odintsov, Phys. Lett. B 562, 147 (2003); Y.H. Wei and Y. Tian, Class. Quant. Grav. 21, 5347 (2004); V.K. Onemli and R. P. Woodard, Phys. Rev. D 70, 107301 (2004); M. R. Setare, Eur. Phys. J. C 50, 991 (2007); M. Jamil, Int. J. Theor. Phys. 49, 144 (2010); ibid, Int. J. Theor. Phys. 49, 62 (2010); ibid, Eur. Phys. J. C 62, 609 (2009); M. Jamil et al, Phys. Lett. B 679, 172 (2009); ibid, Int. J. Theor. Phys. 49, 42 (2010); ibid, Eur. Phys. J. C 61, 471 (2009); ibid, arXiv:1002.1434v1 [gr-qc].

[6] B. Ratra and P.J.E. Peebles, Phys. Rev. D 37, 3406 (1988); C. Wetterich, Nucl. Phys. B 302, 668 (1988); R.R. Caldwell et al, Phys. Rev. Lett. 80, 1582 (1998); I. Zlatev et al, Phys. Rev. Lett. 82, 896 (1999).

[7] C. Armendariz-Picon et al, Phys. Rev. Lett. 85, 4438 (2000) [astro-ph/0004134].

[8] A. Sen, JHEP 0207, 065 (2002);

T. Padmanabhan, Phys. Rev. D 66, 021301 (2002).

[9] N. Arkani-Hamed et al, JHEP 0405, 074 (2004). 
[10] F. Piazza and S. Tsujikawa, JCAP 0407, 004 (2004).

[11] B. Feng et al, Phys. Lett. B 607, 35 (2005); Z. K. Guo et al, Phys. Lett. B 608, 177 (2005); X. Zhang, Commun. Theor. Phys. 44, 762 (2005); A. Anisimov et al, JCAP 0506, 006 (2005); M. R. Setare et al, Phys. Lett. B 660, 299 (2008); J. Sadeghi et al, Phys. Lett. B 662, 92 (2008); M.R. Setare and E.N. Saridakis, Phys. Lett. B 668, 177 (2008); M.R. Setare and E.N. Saridakis, [arXiv:0807.3807 [hep-th]]; M.R. Setare and E.N. Saridakis, JCAP 09, 026 (2008).

[12] J.D. Bekenstein, Phys. Rev. D 7, 2333 (1973); J.D. Bekenstein, Phys. Rev. D 9, 3292 (1974); J.D. Bekenstein, Phys. Rev. D 23, (1981); J.D. Bekenstein, Phys. Rev. D 49, 1912 (1994); S.W. Hawking, Commun. Math. Phys. 43, 199 (1975) [Erratum-ibid. 46, 206 (1976)]; S.W. Hawking, Phys. Rev. D 13, 191 (1976).

[13] A.G. Cohen, D.B. Kaplan, and A.E. Nelson, Phys. Rev. Lett. 82, 4971 (1999).

[14] M. Alimohammadi and H. Behnamian, Phys. Rev. D 80, 063008 (2009).

[15] V. Faraoni, Phys. Rev. D 80, 124040 (2009).

[16] M. Allahverdizadeh et al, Phys. Rev. D 81, 044001 (2010).

[17] C.H. Brans and R.H. Dicke, Phys. Rev. 124, 925 (1961); C.H. Brans, Phys. Rev. 125, 2194 (1962).

[18] B. Bertotti et al, Nature 425, 374 (2003); J.P. de Leon, arXiv:1001.1961v1 [gr-qc]; S. Sen and A.A. Sen, Phys. Rev. D 63, 124006 (2001).

[19] M. R. Setare, Phys. Lett. B 644, 99 (2007).

[20] C-J. Feng, arXiv:0806.0673v1 [hep-th]; C-Y. Chena and Y-G. Shen, arXiv:hepth/0312225v2

[21] T. Clifton and J.D. Barrow, Phys. Rev. D 73, 104022 (2006).

[22] M.H. Dehghani et al, Phys. Lett. B 659, 476 (2008); Y.S. Myung et al, Mod. Phys. Lett. A 23, 91 (2008).

[23] S. Das and N. Banerjee, Phys. Rev. D 78, 043512 (2008).

[24] Y. Gong, Phys. Rev. D 70, 064029 (2004).

[25] B. Bertotti et al, Nature 425374 (2003); V. Acquaviva and L. Verde, JCAP 12001 (2007).

[26] S.D.H. Hsu, Phys. Lett. B 594, 13 (2004).

[27] M. Li, Phys. Lett. B 603, 1 (2004).

[28] M.C. Bento et al, Phys. Rev. D 66, 043507 (2002); M.C. Bento et al, Phys. Rev. D 70, 083519 (2004); R.A. Sussman, Phys. Rev. D 79, 025009 (2009).

[29] V. Gorini et al, JCAP 0802, 016 (2008).

[30] H. Kim et al, Phys. Lett. B 628, 11 (2005); N. Banerjee and D. Pavon, Phys. Lett. B 647, 477 (2007).

[31] A. Sheykhi, Phys. Rev. D 81, 023525 (2010).

[32] M. Li et al, arXiv:0912.3988 [astro-ph.CO]

[33] H. Wei, Commun. Theor. Phys. 52, 743 (2009). 